\title{
Hard versus soft dynamics for adsorption-desorption kinetics: Exact results in one-dimension
}

\author{
S. J. Manzi, V. J. Huespe, R. E. Belardinelli, and V. D. Pereyra \\ Departamento de Física, Instituto de Física Aplicada (INFAP)_CONICET, Universidad Nacional de San Luis, Chacabuco 917, \\ 5700 San Luis, Argentina
}

(Received 15 April 2009; revised manuscript received 18 September 2009; published 12 November 2009)

\begin{abstract}
The adsorption-desorption kinetics is discussed in the framework of the kinetic lattice-gas model. The master equation formalism has been introduced to describe the evolution of the system, where the transition probabilities are written as an expansion of the occupation configurations of all neighboring sites. Since the detailed balance principle determines half of the coefficients that arise from the expansion, it is necessary to introduce ad hoc, a dynamic scheme to get the rest of them. Three schemes of the so-called hard dynamics, in which the probability of transition from single site cannot be factored into a part which depends only on the interaction energy and one that only depends on the field energy, and five schemes of the so-called soft dynamics, in which this factorization is possible, were introduced for this purpose. It is observed that for the hard dynamic schemes, the equilibrium and nonequilibrium observables, such as adsorption isotherms, sticking coefficients, and thermal desorption spectra, have a normal or physical sustainable behavior. While for the soft dynamics schemes, with the exception of the transition state theory, the equilibrium and nonequilibrium observables have several problems. Some of them can be regarded as abnormal behavior.
\end{abstract}

DOI: 10.1103/PhysRevE.80.051112 PACS number(s): 02.50.-r, 68.43.Mn, 68.43.Nr, 47.11.Qr

\section{INTRODUCTION}

The study of surfaces and interfaces is of fundamental importance in the understanding of different processes in nature and in a wide variety of technological applications, such as heterogeneous catalysis, electronic, magnetic, and optical devices, sensors, coatings and many industrial systems and processes [1,2]. The kinetic behavior of the gas-solid interfaces is currently one of the most interesting and well referenced phenomena in surface science [3-18].

The kinetic lattice-gas model (KLGM) is the most adequate tool for the treatment of the kinetics when the adsorbate does not remain in a quasiequilibrium state during the adsorption and desorption processes. The model was developed in close analogy with the time dependent Ising model for magnetic systems, which was originally introduced by Glauber $[19,20]$. In its simplest form the KLGM is restricted to the submonolayer regime and to the gas-solid system where the surface structure and the adsorption sites do not change with the coverage [21]. However, further generalizations of the KLGM have been made in the past for several and different problems [18,22-27].

The KLGM is based in the master equation approach where adsorption, desorption, diffusion, and other phenomena are introduced as Markovian processes through transition probabilities, which must satisfy the principle of detailed balance (PDB).

Despite the fact that, detailed microscopic mechanisms of the surface processes are usually not known, the transition probabilities can be written in terms of the occupation configurations of all neighboring sites in the KLGM $[9,17,18,21-24,28,29]$. In this point, the PDB imposes a set of restrictions on the coefficients of adsorption $A_{i}$, desorption $D_{i}$, and diffusion $C_{i}$. This has been discussed in Refs. $[17,18,21,24,30]$, where the authors have introduced different kinetics, according to the relations among those coefficients.
On the other hand, it is well known that different microscopic dynamics can yield different equilibrium paths and equilibrium fluctuations [31] (cluster versus local Monte Carlo (MC) algorithms being the most extreme examples [32]) and even noticeable differences in the steady-state microstructure $[33,34]$. Nevertheless, the general expectation is that, if no additional parameters (such as an activation barrier or a diffusion rate) are introduced into the physical model, the observables are only affected quantitatively.

Recent studies indicate that different stochastic dynamics lead to important differences in the nanostructure of fielddriven interfaces [35-39] even when they have the same conserved quantities and satisfy PDB.

Before defining the transition probabilities, an important distinction must be made between models with hard dynamics [37,39-44], in which the single-site transition rates cannot be factorized into one part that depends only on the interaction energy and another that depends only on the field energy, in contrast with those models with soft dynamics $[36,38,39,45-52]$, for which this factorization is possible.

In this context, Kang et al. [49] analyzed different choices for the transition probabilities in MC simulations for studying the growth exponent in the growth of domains. They showed that the choice of transition probabilities affects directly the dynamic quantities. Particularly, they found this dependence on the growth exponent in the ordered domains out of equilibrium [53].

In the same way, Rikvold et al. showed that the intrinsic interface width and properties in field-driven solid-on-solid interface studies depend on the choice of dynamics. They have found that, in the framework of the soft dynamics, all dependences on the field are canceled due to the PDB $[36,38,39]$. On the other hand, in hard dynamics the intrinsic interface width and properties, such as the propagation velocity, are strongly affected by the field [36].

Recently, different dynamic schemes have been introduced to analyze the adsorption-desorption kinetics in the framework of the one-dimensional KLGM [30]. 
The interaction energy between the particle and the surface depends on the distance between them. Therefore a particle near the surface experiences the same interaction energy during the adsorption or desorption processes. As a consequence of this statement, in a one-dimensional system with nearest neighbor lateral interaction, one should obtain: (i) for attractive lateral interaction the adsorption isotherms growth monotonically without steps, the corresponding normalized sticking coefficient must be higher than $1-\theta$ growing with the lateral interaction and the temperature programmed desorption (TPD) spectrum must present only one peak shifting to higher temperatures for increasing interaction; (ii) for repulsive lateral interaction the adsorption isotherms must be present a step at half coverage as one lowers the temperature, the corresponding normalized sticking coefficient must be lower than $1-\theta$ decreasing with the higher lateral interactions and the TPD spectrum must present three peaks for immobile adsorbate and two peaks for mobile adsorbates for low enough temperatures and high initial coverages. According to that, any other behavior could be considered as anomalous. In Ref. [30] the authors have considered additional constrains on the adsorption and desorption coefficients. They have demonstrated that a linear relation between those coefficients leads to anomalous behavior in the equilibrium and nonequilibrium observables, while the use of the transition state theory (TST) $[8,14]$ leads to a correct behavior without physical inconsistencies. However, a detailed analysis of the influence of the hard and soft dynamics on the behavior of the adsorption-desorption kinetics has not been reported yet.

The aim of the present paper is to study the influence of different dynamic schemes in the behavior of the adsorptiondesorption kinetics. It is observed that for five different scenarios of soft dynamics and for three examples of hard dynamics the equilibrium and nonequilibrium observables, such as the adsorption isotherms, sticking coefficients, and thermal desorption spectra present different behaviors (some of them anomalous).

The outline of the paper is as follows: in Sec. II the KLGM is set up, the master equation is introduced and the transition probabilities are expanded in terms of the occupation configurations of all neighboring sites. In Sec. III, the observables such as the adsorption isotherms, the sticking coefficients, and the TPD spectra are introduced. The general trends for each of them are discussed. In Sec. IV the dynamic schemes are introduced and the influence on the kinetic observables is discussed. Finally, in Sec. V the conclusions are given.

\section{KINETIC LATTICE GAS MODEL AND THE MASTER EQUATION}

To set up the KLGM, one restricts the analysis to a gassolid system in which all relevant processes, such as diffusion, adsorption, desorption, reactions, etc., are Markovian. One assumes that the system can be divided into cells, labeled $i$, for which one introduces microscopic variables $n_{i}$ $=1$ or 0 depending on whether cell $i$ is occupied by an adsorbed gas particle or not. To introduce the dynamics of the system is written down a model Hamiltonian

$$
H=E_{s} \sum_{i} n_{i}+\frac{1}{2} V \sum_{\langle i j\rangle} n_{i} n_{j}+\cdots .
$$

Here $E_{s}$ is a single particle adsorption energy and $V$ is the two particle interaction between nearest neighbors $\langle i j\rangle$. Interactions between next-nearest neighbors, etc. and many particle interactions can be easily added to Eq. (1).

As long as the number of particles in the adsorbate does not change, which is the case for systems in equilibrium or diffusion studies, the first term in Eq. (1) is constant and can be dropped from further consideration. However, if the goal is the study of the adsorption-desorption kinetics, the number of particles in the adsorbate changes as a function of time and a proper identification of $E_{S}$ is mandatory. Arguing that the lattice-gas Hamiltonian should give the same Helmholtz free energy as a microscopic Hamiltonian (for noninteracting particles) one can show that the proper identification is given by $[54]$

$$
E_{s}=-V_{0}-\frac{1}{\beta} \ln \left(q_{3} q_{\text {int }}\right)-\frac{1}{\beta}\left[\ln \left(\beta \lambda^{3} P\right)-\ln \left(Z_{\text {int }}\right)\right],
$$

where $\beta=1 / k_{B} T ; k_{B}$ and $T$ are the Boltzmann constant and the absolute temperature, respectively. $V_{0}$ is the (positive) depth of the surface potential, $q_{3}$ is the single particle partition function of an adsorbed particle, $q_{\text {int }}$ is the internal partition function for (frustrated) vibrations and rotations of the adsorbed molecule, $P$ is the pressure in the gas phase above the surface, and $\lambda=h / \sqrt{2 \pi m k_{B} T}$ is the thermal wavelength of the adparticle with mass $m$ which partition function for (free) vibrations and rotations is $Z_{\text {int }}$.

A function $P(\mathbf{n}, t)$ which gives the probability that a given microscopic configuration $\mathbf{n}=\left(n_{1}, n_{2}, \ldots, n_{N}\right)$ exists at time $t$ is introduced, where $N$ is the total number of adsorption sites on the surface. It satisfies a master equation

$$
\frac{d P(\mathbf{n} ; t)}{d t}=\sum_{n^{\prime}}\left[W\left(\mathbf{n} ; \mathbf{n}^{\prime}\right) P\left(\mathbf{n}^{\prime} ; t\right)-W\left(\mathbf{n}^{\prime} ; \mathbf{n}\right) P(\mathbf{n} ; t)\right],
$$

where $W\left(\mathbf{n}^{\prime} ; \mathbf{n}\right)$ is the transition probability that the microstate $\mathbf{n}$ changes into $\mathbf{n}^{\prime}$ per unit time. It satisfies detailed balance

$$
W\left(\mathbf{n}^{\prime} ; \mathbf{n}\right) P_{0}(\mathbf{n})=W\left(\mathbf{n} ; \mathbf{n}^{\prime}\right) P_{0}\left(\mathbf{n}^{\prime}\right),
$$

where

$$
P_{0}(\mathbf{n})=Z^{-1} \exp [-\beta H(\mathbf{n})],
$$

is the equilibrium probability and $Z$ is the partition function given by

$$
Z=\sum_{\{\mathbf{n}\}} e^{-\beta H(\mathbf{n})} .
$$

In principle, $W\left(\mathbf{n}^{\prime} ; \mathbf{n}\right)$ must be calculated from a Hamiltonian that includes, in addition to Eq. (1), coupling terms to the gas phase and the solid that mediate mass and energy exchange. However, depending of the system, different expressions for the transition probabilities can be proposed. In the transition dynamic approximation (TDA) [46,50], transition rates can- 
not be factorized into one part that depends only on the interaction energy and another that depends only on the field energy [39] (hard dynamics [40]).

Usually, the procedure introduced by Glauber is followed and guesses of an appropriate form for $W\left(\mathbf{n}^{\prime} ; \mathbf{n}\right)$ are made. It is further assumed that the duration of an individual transition, e.g., hopping to a neighboring site, is much shorter than the residence time in the initial state. In this situation there will be only one transition at any given time and the total transition probability can be written as a sum of individual terms.

In order to analyze the simplest cases, let us consider the one-dimensional lattice gas with nearest-neighbor interactions where only direct adsorption and desorption processes are taken into account (no other process is considered). Therefore, the transition probability can be written as

$$
\begin{aligned}
W_{a d-d e s}\left(\mathbf{n}^{\prime} ; \mathbf{n}\right)= & \sum_{i}\left\{w _ { a } ( 1 - n _ { i } ) \left[A_{0}+A_{1}\left(n_{i-1}+n_{i+1}\right)\right.\right. \\
& \left.+A_{2} n_{i-1} n_{i+1}\right]+w_{d} n_{i}\left[D_{0}+D_{1}\left(n_{i-1}+n_{i+1}\right)\right. \\
& \left.\left.+D_{2} n_{i-1} n_{i+1}\right]\right\} \delta\left(n_{i}^{\prime}, 1-n_{i}\right) \Pi_{j \neq i} \delta\left(n_{j}^{\prime}, n_{j}\right) .
\end{aligned}
$$

Here adsorption into site $i$ occurs if initially $n_{i}=0$, with a rate controlled by prospective neighbors if $A_{i} \neq 0$. The Kronecker delta for sites $j \neq i$ excludes multiple transitions.

The equation of motion for the coverage can be obtained by multiplying the master equation by the occupation number $n_{i}$ and summing over all sites. A similar procedure can be used for higher correlation functions [10-12,25,29,55]. Here and elsewhere, some intermediate factorizations and manipulations of correlators are conveniently carried out in diagrammatic form. For instance, the coverage is defined as

$$
\theta(t)=\langle\bullet\rangle=N^{-1} \sum_{i} \sum_{\mathbf{n}} n_{i} P(\mathbf{n} ; \mathbf{t}) .
$$

Similarly,

$$
\langle\circ\rangle=N^{-1} \sum_{i} \sum_{\mathbf{n}}\left(1-n_{i}\right) P(\mathbf{n} ; \mathbf{t})
$$

gives the probability that a site is empty and

$$
\langle\bullet \circ\rangle=\langle\bullet\rangle-\langle\bullet \bullet\rangle=N^{-1} \sum_{i} \sum_{\mathbf{n}}\left(1-n_{i}\right) n_{i+1} P(\mathbf{n} ; \mathbf{t})
$$

is the conditional probability that out of two neighboring sites one is empty and one is occupied.

After some straightforward calculations the following expressions for the correlators can be obtained [30]:

$$
\begin{gathered}
\frac{d \theta}{d t}=w_{a}\left[Q_{a d s}^{0}\langle\circ \circ \circ\rangle+2 Q_{a d s}^{1}\langle\bullet \circ \circ\rangle+Q_{a d s}^{2}\langle\bullet \circ \bullet]-w_{d}\left[Q_{d e s}^{0}\langle\circ \bullet\right.\right. \\
\left.\circ\rangle+2 Q_{d e s}^{1}\langle\bullet \bullet\rangle+Q_{d e s}^{2}\langle\bullet \bullet\rangle\right], \\
\left.\frac{d\langle\bullet \bullet\rangle}{d t}=2 w_{a}\left[Q_{a d s}^{1}\langle\bullet \circ\rangle\right\rangle+Q_{a d s}^{2}\langle\bullet \circ\rangle\right]-2 w_{d}\left[Q_{d e s}^{1}\langle\bullet \bullet\rangle\right. \\
+Q_{d e s}^{2}\langle\bullet \bullet \bullet],
\end{gathered}
$$

$$
\begin{aligned}
\frac{d\langle\bullet \circ\rangle}{d t}= & w_{a}\left[2 Q_{a d s}^{0}\langle\bullet \circ \circ \circ\rangle+2 Q_{a d s}^{1}\left\langle\bullet \circ \circ \bullet-Q_{a d s}^{2}(\langle\bullet \circ \bullet \bullet\rangle\right.\right. \\
& +\langle\bullet \circ \bullet \circ)]-w_{d}\left[2 Q_{d e s}^{0}\langle\bullet \circ \bullet \circ\rangle+2 Q_{d e s}^{1}\langle\bullet \circ \bullet \bullet\rangle\right. \\
& -Q_{d e s}^{2}(\langle\bullet \bullet \circ\rangle+\langle\bullet \bullet \bullet)], \\
\frac{d\langle\bullet \bullet\rangle}{d t}= & w_{a}\left[2 Q_{a d s}^{1}\langle\bullet \bullet \circ\rangle+Q_{a d s}^{2}(\langle\bullet \circ \bullet \circ\rangle+3\langle\bullet \circ \bullet\rangle)\right] \\
& -w_{d}\left[2 Q_{d e s}^{1}\langle\bullet \bullet \circ\rangle+Q_{d e s}^{2}(\langle\bullet \bullet \circ\rangle+3\langle\bullet \bullet \bullet\rangle)\right] .
\end{aligned}
$$

The PDB imposes a set of restrictions on the coefficients $A_{i}$ and $D_{i}$ [24], which are the following:

$$
\begin{gathered}
w_{a} A_{0}=w_{d} D_{0} e^{-\beta E_{s}}, \\
w_{a}\left(A_{0}+A_{1}\right)=w_{d}\left(D_{0}+D_{1}\right) e^{-\beta\left(E_{s}+V\right)},
\end{gathered}
$$

and

$$
w_{a}\left(A_{0}+2 A_{1}+A_{2}\right)=w_{d}\left(D_{0}+2 D_{1}+D_{2}\right) e^{-\beta\left(E_{s}+2 V\right)} .
$$

$w_{a}$ and $w_{d}$ cannot be fixed by detailed balance because they contain the information about the energy exchange with the solid in the adsorption and desorption processes, which is not in the static lattice-gas Hamiltonian [24]. However, if one consider that $w_{a}=w_{d}=w_{0}$, and by comparison with the phenomenological expression for adsorption one can identify it as

$$
w_{0}=S_{0}(T) \frac{P \lambda a_{s}}{h},
$$

where $S_{0}(T)$ is the temperature-dependent sticking coefficient at zero coverage and $a_{s}$ is the area of a surface unit cell. It contains the dynamic information about the energy transfer from the adsorbing particle to the solid which gives rise to its temperature dependence, for instance, an exponential Boltzmann factor for activated adsorption. It can be calculated only on the basis of a dynamic theory that accounts for the coupling of the adparticles to the vibrational and electronic degrees of freedom of the substrate and must be postulated ad hoc within the context of the kinetic lattice-gas model.

The long-range interaction introduces similar constraints on the other coefficients. Each of such constraint introduces two new coefficients. However, detailed balance provides only half the number of relations to fix these unknown coefficients in the transition probabilities. Again, the static (lattice-gas) Hamiltonian cannot completely dictate the kind of kinetics possible in the system. As it is pointed out in Refs. $[17,21,24]$, any functional relation between the $A$ and $D$ coefficients must be postulated $a d$ hoc or calculated from a microscopic Hamiltonian that accounts for coupling of the adsorbate to the lattice or electronic degrees of freedom of the substrate.

In order to get new conditions on the coefficients, note that $\langle\circ \circ \circ\rangle,\langle\bullet \circ \circ\rangle,\langle\bullet \circ \bullet\rangle,\langle\circ \bullet \circ\rangle,\langle\bullet \bullet\rangle$, and $\langle\bullet \bullet\rangle$, as well as $\langle\bullet$ $\circ \circ \circ\rangle,\langle\bullet \bullet \circ \bullet\rangle,\langle\bullet \bullet \bullet\rangle,\langle\bullet \circ \bullet\rangle\rangle,\langle\bullet \bullet \circ\rangle,\langle\bullet \bullet \bullet\rangle$, and $\langle\bullet \bullet \circ\rangle$ are mutually exclusive conditional probabilities that lie between 0 and 1 . Besides that, those quantities that multiply the con- 
ditional probabilities in Eqs. (11)-(14) should be interpreted as rates and must be non-negative. After some calculations one can obtain the following relations [30]

$$
\begin{gathered}
Q_{a d s}^{0}=A_{0} \geq 0, \\
Q_{a d s}^{1}=\left(A_{0}+A_{1}\right) \geq 0, \\
Q_{a d s}^{2}=\left(A_{0}+2 A_{1}+A_{2}\right) \geq 0, \\
Q_{d e s}^{0}=D_{0} \geq 0, \\
Q_{d e s}^{1}=\left(D_{0}+D_{1}\right) \geq 0,
\end{gathered}
$$

and

$$
Q_{\text {des }}^{2}=\left(D_{0}+2 D_{1}+D_{2}\right) \geq 0 .
$$

These inequalities must be fulfilled in addition to the PDB in order for the dynamic yield correct results.

\section{DESCRIPTION OF THE OBSERVABLES}

In this section the observables, such as adsorption isotherms, sticking coefficients, and (TPD) spectra, are described. The general trend for each of them is discussed. To make that the set of coupled equations of motion for correlation functions be the basis of an analytic theory of surface processes, the hierarchy must be truncated. Several works have been written about closure approximations, particularly
ben-Avraham and Köhler in Ref. [56] considered a meanfield $(n, m)$-cluster approximation for different lattice models. The simplest closure approximation is the Kirkwood approximation in which all higher correlation functions are expressed in terms of two-body correlation functions. Therefore, in equilibrium the equations of motion for the coverage and the two-site nearest-neighbor correlation function are retained. On two-dimensional lattices the two-site closure yields the quasichemical approximation which is exact on a one-dimensional lattice. However, away from equilibrium this closure scheme no longer holds exactly.

\section{A. Adsorption isotherms}

To obtain the exact expression for the adsorption isotherm and two-body correlation function, after using a closure scheme, Eqs. (11) and (12) must be set equal to zero $[25,30,55]$. These expressions depend on the adsorption and desorption coefficients. However, when both the principle of detail balance as the relations given in Eqs. (19)-(24) are fulfilled, the expression for the chemical potential is given by

$$
e^{\beta \mu}=\frac{\theta^{2}+2 \theta\left(e^{\beta V}-1\right)\langle\bullet \bullet\rangle+\left(e^{\beta V}-1\right)^{2}\langle\bullet \bullet\rangle^{2}}{\theta(1-\theta)}
$$

or equivalently

$$
e^{\beta \mu}=\frac{\alpha-1+2 \theta}{\alpha+1-2 \theta} e^{\beta V},
$$

and the two-body correlation function is

$$
\langle\bullet \bullet\rangle=\frac{\left(e^{\beta V}-2\right) \theta-e^{\beta V}(1-\theta)+\sqrt{e^{2 \beta V} \theta^{2}+e^{\beta V}(1-\theta)\left(e^{\beta V}(1-3 \theta)+4 \theta\right)}}{2\left(e^{\beta V}-1\right)}
$$

or

$$
\langle\bullet\rangle=\theta\left[1-2 \frac{1-\theta}{1+\alpha}\right],
$$

with

$$
\alpha=\sqrt{1-4 \theta(1-\theta)\left(1-e^{-\beta V}\right)} .
$$

These equations represent the functional relation among the coverage, two-site correlation function, and the chemical potential provided that the detailed balance principle and Eqs. (19)-(24) are fulfilled.

\section{B. Sticking coefficient}

The sticking coefficient is a measure of the efficiency of the energy transfer in the adsorption and desorption processes. Therefore, it cannot be obtained from thermodynamic arguments but it must be either calculated from a microscopic theory or postulated in a phenomenological approach based on experimental evidence for a particular system or some simple arguments.

In order to calculate the normalized sticking coefficient the following definition is used [18]:

$$
S(\theta, T)=A_{0}\langle\circ\rangle+2 A_{1}\langle\bullet \circ\rangle+A_{2}\langle\bullet \circ \bullet\rangle .
$$

For a system in which surface diffusion is much faster than adsorption, the adsorbate remains in quasiequilibrium during adsorption so that the equilibrium expressions and properties of the correlators can be used [55]. Also, a $(2,1)$ cluster approximation is used to factorize the three-site correlators in Eq. (30). In this context, the expressions for these correlators are the following:

$$
\langle\bullet \circ\rangle=\frac{\langle\bullet \circ\rangle^{2}}{\langle\circ\rangle}
$$




$$
\langle\bullet \circ\rangle=\frac{2 \theta(1-\theta)}{1+\alpha} .
$$

Next, the sticking coefficient is calculated in the limits of null, large attractive, and large repulsive interactions.

(i) For null interaction, $V=0$, the particle-hole correlator is exactly given by $\langle\bullet \bullet\rangle=\theta(1-\theta)$ and similarly $\langle\bullet \bullet \bullet\rangle=\theta^{2}(1$ $-\theta)$. Then, the normalized sticking coefficient can be written as

$$
\frac{S(\theta, T)}{S(0, T)}=(1-\theta)\left(1+2 \frac{A_{1}}{A_{0}} \theta+\frac{A_{2}}{A_{0}} \theta^{2}\right) .
$$

(ii) For large attraction, $\beta V \ll 0$, the expression for the normalized sticking coefficient must be written as

$$
\frac{S(\theta, T)}{S(0, T)}=(1-\theta)+2 \frac{A_{1}}{A_{0}}\langle\bullet \circ\rangle+\frac{A_{2}}{A_{0}} \frac{(\langle\bullet \circ\rangle)^{2}}{(1-\theta)} .
$$

Due to the fact that the particle-hole correlator tends to zero as $e^{\beta V / 2}$ increases, the limits in the second and third terms in the rhs of Eq. (34) must be considered for each dynamic scheme in particular.

(iii) For large repulsion, $\beta V \gg 0$, one can expand the particle-hole correlator in power series of the factor $\left(1-e^{-\beta V}\right)$ to the first order, distinguishing two cases: for $\theta<0.5$,

$$
\frac{\langle\bullet \bullet\rangle}{\langle\circ\rangle} \simeq \frac{\theta}{(1-\theta)}\left[1-\frac{\theta}{(1-2 \theta)} e^{-\beta V}\right],
$$

then, the normalized sticking coefficient can be written as

$$
\frac{S(\theta, T)}{S(0, T)}=(1-\theta)+2 \frac{A_{1}}{A_{0}} \theta+\frac{A_{2}}{A_{0}} \frac{\theta^{2}}{(1-\theta)},
$$

while for $\theta>0.5$,

$$
\frac{\langle\bullet \circ\rangle}{\langle\circ\rangle} \simeq 1-\left(\frac{\theta-1}{2 \theta-1}\right) e^{-\beta V},
$$

then, the normalized sticking coefficient is given by

$$
\frac{S(\theta, T)}{S(0, T)}=(1-\theta)\left(1+2 \frac{A_{1}}{A_{0}}+\frac{A_{2}}{A_{0}}\right) .
$$

As for the attractive case, the limits are calculate to take into account each dynamic scheme in detail. In all the former cases, $S(0, T)=A_{0}$ is assumed [30].

\section{Thermal desorption spectra}

To analyze the desorption process it is necessary to go beyond the two-site closure scheme. It is well known [55] that a $(3,2)$-cluster approximation is enough to obtain the exact solution of the TPD spectra in absence of diffusion. All higher-order correlation functions will be factorized in terms of three-site correlation functions in the numerator with twosite overlap. After that, the TPD spectra are obtained from the solution for the first four correlations [Eqs. (11)-(14)] where the adsorption terms are neglected [25,55,57].

Although the diffusion process is not taken into account in the present work, it is well established that, for very fast diffusion, the desorption kinetic are known exactly because the adsorbate remains in quasiequilibrium throughout desorption. In this way, the desorption rate is given by [58]

$$
\frac{d \theta}{d t}=-S(\theta, T) a_{s} \frac{2 \pi m k_{B}^{2} T^{2}}{h^{3}} \exp \left[\mu(\theta, T) / k_{B} T\right] .
$$

The TPD spectrum resulting from this phenomenological equation and the spectrum obtained from the solution of the kinetic equations with the inclusion of the diffusion terms in the limit of high mobility are in complete agreement $[25,55]$. Then, the phenomenological equation [Eq. (39)] is more practical in order to obtain mobile TPD spectra.

In all TPD spectra the desorption is considered as an activated process, where the activation energy is $10 \mathrm{kcal} / \mathrm{mol}$, the pre-exponential factor is $10^{13} \mathrm{~s}^{-1}$ and the initial coverage is $\theta_{0}=0.95$. In the last equation $m=40$ u.m.a and $a_{s}$ $=10 \AA^{2}$ is considered.

In what follows the different dynamic schemes are introduced and a detailed analysis of the consequences on the adsorption isotherms, sticking coefficients, and TPD curves will be done.

\section{DYNAMIC SCHEMES AND THE BEHAVIOR OF THE OBSERVABLES}

In this section, different dynamic schemes are introduced by means of defining the transition probabilities. Then, the adsorption $\left(A_{i}\right)$ and desorption $\left(D_{i}\right)$ coefficients are obtained and used to calculate the adsorption isotherms, the sticking coefficients, and the TPD spectra.

As it pointed out in the introduction, two different dynamic schemes can be distinguished. The first one corresponds to those models with hard dynamics in which the single-site transition rates cannot be factorized into one part that depends only on the interaction energy and another that depends only on the field energy. The second corresponds to those models with soft dynamics, for which this factorization is possible.

In what follows we introduce the different schemes for a one-dimensional lattice gas with nearest-neighbor lateral interaction. The behavior of the observables is also obtained for each dynamic scheme. In particular, in case of the normalized sticking coefficient, the limits for large lateral interaction (attractive and repulsive) are calculated.

\section{A. Ising kinetics (hard dynamics)}

The Ising kinetics has been discussed, in the framework of the two-dimensional KLGM by Kreuzer and Zhang [21] and is used in most papers that deal with surfaces in the spin language [59-62]. Originally, the authors have introduced the following relations between the coefficients instead of the transition probabilities;

$$
\begin{gathered}
A_{0}=1, \\
A_{i}=\gamma D_{i},
\end{gathered}
$$

with $i=1,2$. The last relations together with PDB lead to 


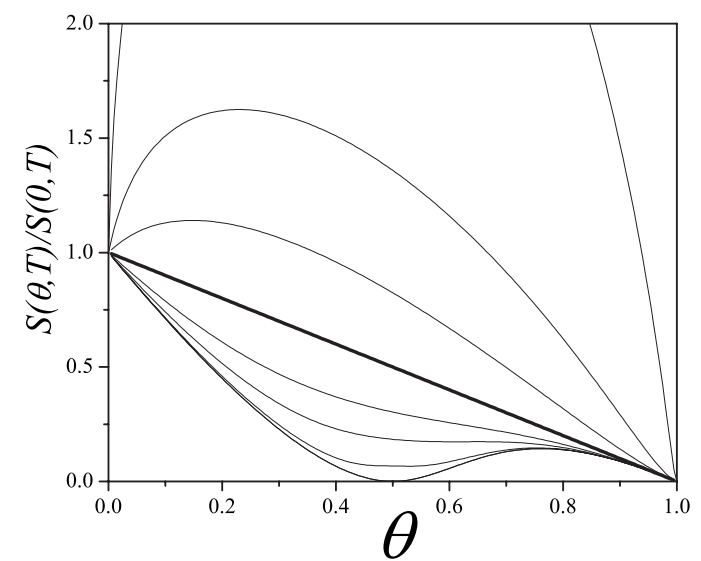

FIG. 1. Normalized sticking coefficient for Ising kinetics with $\gamma=-1$ and different values of the lateral interaction. Top to bottom: $\beta V=-4,-2,-1,0,1,2,4,20$.

$$
\begin{gathered}
D_{0}=e^{\beta E_{S}}, \\
D_{1}=D_{0}\left(\frac{e^{\beta V}-1}{1-\gamma D_{0} e^{\beta V}}\right), \\
D_{2}=D_{0}\left(\frac{e^{2 \beta V}-1}{1-\gamma D_{0} e^{2 \beta V}}\right)-2 D_{1} .
\end{gathered}
$$

These relations are equivalent to the following transition probabilities:

$$
W_{a d s}^{i}=\frac{1-\gamma e^{\beta E_{S}}}{1-\gamma e^{\beta\left(E_{S}+i V\right)}}
$$

and

$$
W_{d e s}^{i}=\frac{1-\gamma e^{\beta E_{S}}}{e^{-\beta\left(E_{S^{+}}+i\right)}-\gamma} .
$$

The last two equations must be positive according to Eqs. (19)-(24). Therefore, $\gamma<0$ for any value of lateral interactions. When $\gamma>0$, the adsorption isotherms present an anomalous behavior.

The normalized sticking coefficient for the Ising kinetics is shown in Fig. 1. As is observed, the sticking coefficient grows with the increasing attractive lateral interaction. This is because for large values of attractive interaction, $\beta V \ll 0$, both terms, $\left[A_{1}\langle\bullet \circ\rangle / A_{0}\right]$ and $\left[A_{2}(\langle\bullet \circ\rangle)^{2} /\left(A_{0}(1-\theta)\right)\right]$ diverge as $e^{-\beta V / 2}$ and $e^{-\beta V}$ increase. As expected, all the curves drop to zero at monolayer coverage [see Eq. (30)].

For large repulsive lateral interaction and for coverage $\theta<1 / 2$, the normalized sticking coefficient is given by

$$
\frac{S(\theta, T)}{S(0, T)} \rightarrow 1-3 \theta+\frac{\theta^{2}}{(1-\theta)} .
$$

This occurs because $\left[A_{1}\langle\bullet \circ\rangle / A_{0}\right] \rightarrow-\theta$, while $\left[A_{2}(\langle\bullet \circ\rangle)^{2} /\right.$ $\left.\left(A_{0}(1-\theta)\right)\right] \rightarrow \theta^{2} /(1-\theta)$, when $\beta V \gg 0$.

Note that $S(\theta, T) / S(0, T)$ does not depend on $\gamma$. However, for coverage $\theta>1 / 2$, the term $A_{1} / A_{0} \rightarrow 0$, while the term $A_{2} / A_{0} \rightarrow\left\{\gamma \theta(1-\theta) /\left[(2 \theta-1)^{2}-\gamma \theta(1-\theta)\right]\right\}$ [to calculate those limits it is necessary to considered that $e^{-\beta E_{S}} \rightarrow e^{2 \beta V}(2 \theta$
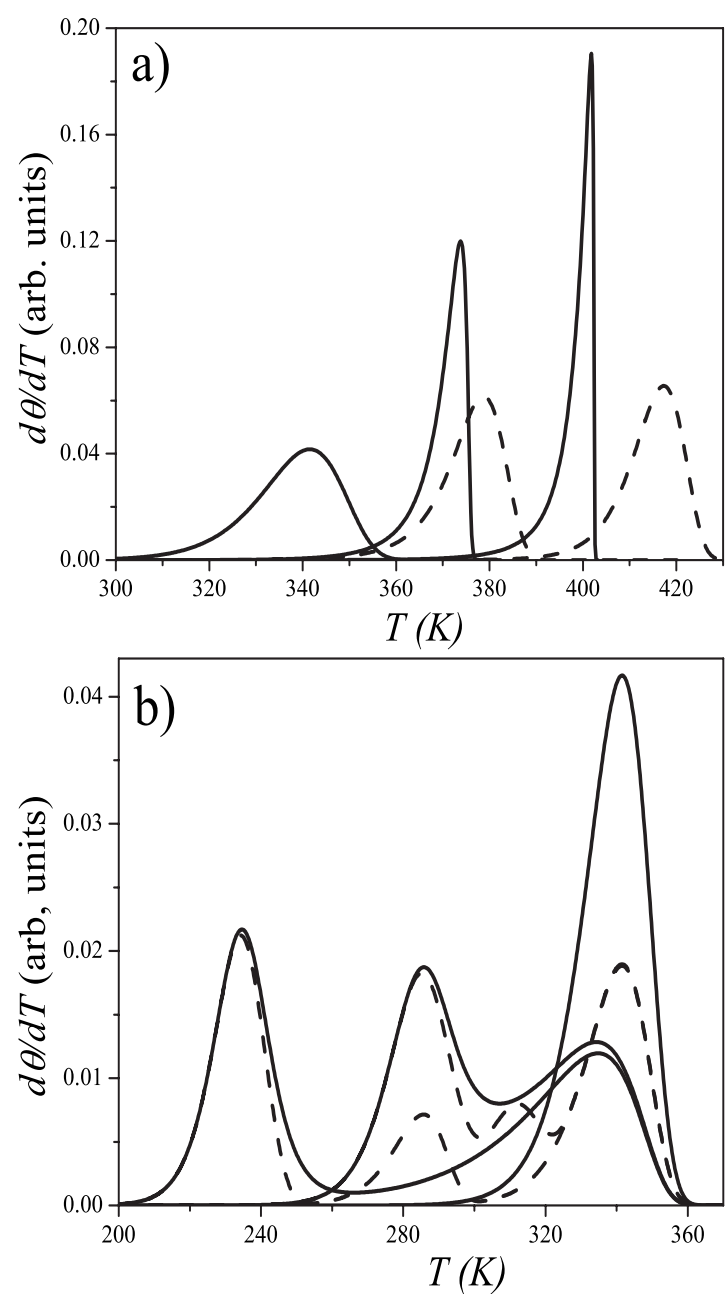

FIG. 2. Thermal desorption spectra using Ising kinetics with $\gamma$ $=-1$ for different lateral interactions: (a) attractive case: left to right, mobile (line) $V(\mathrm{kcal} / \mathrm{mol})=-2,-4$ and immobile (dash) $V(\mathrm{kcal} / \mathrm{mol})=-2,-4$; (b) repulsive case: left to right, mobile (line) $V(\mathrm{kcal} / \mathrm{mol})=4,2$, and immobile (dash) $V(\mathrm{kcal} / \mathrm{mol})=4,2$. The case of null interaction is also included in both figures.

$-1)^{2} / \theta(1-\theta)$ for large attraction, $\beta V \gg 0$, see Eq. (25)]. Then, the expression for the normalized sticking coefficient is given by

$$
\frac{S(\theta, T)}{S(0, T)}=\frac{(1-\theta)(2 \theta-1)^{2}}{(2 \theta-1)^{2}-\gamma \theta(1-\theta)} .
$$

The effect of $\gamma$ on the sticking coefficient can be analyzed as follows: for $\gamma \rightarrow-\infty$, the term $A_{2} / A_{0} \rightarrow-1$. Therefore, the sticking coefficient $S(\theta, T) / S(0, T) \rightarrow 0$; while for $\gamma \rightarrow 0$, the term $A_{2} / A_{0} \rightarrow 0$ and consequently the sticking coefficient $S(\theta, T) / S(0, T) \rightarrow(1-\theta)$.

The immobile and mobile TPD spectra, for both attractive and repulsive lateral interactions, are shown in Figs. 2(a) and 2(b), respectively. In the first case, the temperature of the peak corresponding to the mobile TPD is lower than the temperature of the peak corresponding to the immobile TPD, considering the same lateral interaction. For repulsive lateral interactions, the TPD curves for immobile adsorbate present the three characteristic peaks. The low-temperature peak cor- 


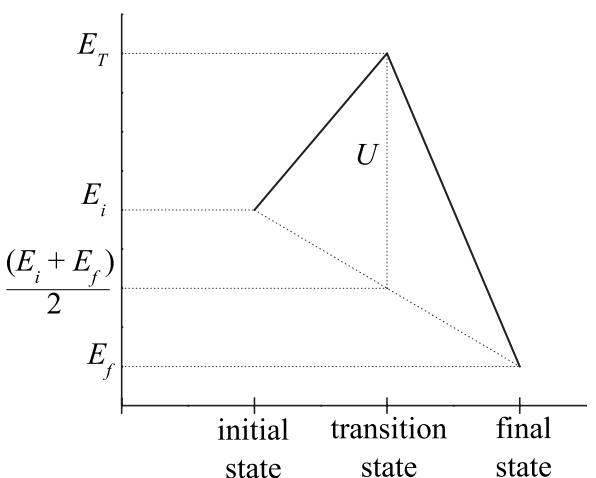

FIG. 3. Schematic picture of the transition barrier in the symmetric Butler-Volmer approximation, used to calculate the TDA and OSD transition rates. After Ref. [46].

responds to desorption of particles which have two neighbors, the middle peak is due to desorption of those particles that have one neighbor, and the last peak corresponds to desorption of isolated particles. On the other hand, mobile TPD curves present only two peaks. The middle peak disappears due to the redistribution of the particles. In both figures, the case $V=0$ is included.

Note that, in Ref. [21], the authors analyzed a twodimensional square lattice with nearest-neighbor interactions. They have used a Kirkwood closure scheme to truncate the rate equations systems. Under these conditions, for very large attractive interactions, the sticking coefficient increases very strongly with coverage. On the other hand, desorption is trivially first order throughout the coverage regime. They have concluded that very few, if any, physical systems show such behavior. However, for one-dimensional system, where exact solution is possible, the Ising kinetics does not present such anomalous behavior provided that the restrictions on $\gamma$ are fulfilled.

\section{B. Two-steps transition dynamic approximation (hard dynamics)}

The two-step TDA [50-52] has been introduced to explain the anomalous diffusion anisotropy of $\mathrm{H}$ adatoms on a $W(110)$ surface and to study the microscopic structure and the stationary propagation velocity of $(1+1)$-dimensional solid-on-solid interfaces in an Ising lattice-gas model [39]. In the TDA scheme the transition probability is defined by

$$
W_{T D A}=\left(\frac{1}{1+e^{\beta\left(E_{T}-E_{i}\right)}}\right)\left(\frac{1}{1+e^{\beta\left(E_{f}-E_{T}\right)}}\right),
$$

with the condition that

$$
E_{T}=\frac{E_{f}+E_{i}}{2}+U
$$

$U$ determines the energy barrier between the two states (see Fig. 3). From the last two equations it is possible to obtain the expression for the transition probabilities for the adsorption process as

$$
W_{a d s}^{i}=\left(\frac{1}{1+e^{\beta\left[\left(E_{S}+i V\right) / 2+U\right]}}\right)\left(\frac{1}{1+e^{\beta\left[\left(E_{S}+i V\right) / 2-U\right]}}\right),
$$

while for the desorption process is

$$
W_{d e s}^{i}=\left(\frac{1}{1+e^{-\beta\left[\left(E_{S}+i V\right) / 2+U\right]}}\right)\left(\frac{1}{1+e^{-\beta\left[\left(E_{S}+i V\right) / 2-U\right]}}\right) .
$$

The expressions for the adsorption and desorption coefficients are given by

$$
A_{i}=\left\{\frac{1}{1+e^{\beta\left[\left(E_{S}+i V\right) / 2+U\right]}}\right\}\left\{\frac{1}{1+e^{\beta\left[\left(E_{S}+i V\right) / 2-U\right]}}\right\}-\sum_{j=0}^{i} j A_{j-1}
$$

and

$$
D_{i}=\left\{\frac{1}{1+e^{-\beta\left[\left(E_{S}+i V\right) / 2+U\right]}}\right\}\left\{\frac{1}{1+e^{-\beta\left[\left(E_{S}+i V\right) / 2-U\right]}}\right\}-\sum_{j=0}^{i} j D_{j-1},
$$

respectively.

The normalized sticking coefficient for the TDA is shown in Fig. 4. For a fixed value of the transition state energy $U$, the behavior of $S(\theta, T) / S(0, T)$ can be explained as follows. As the lateral interaction increases, the normalized sticking coefficient increases monotonically in the range, $0<\theta<1$, dropping to zero at monolayer. This occurs because the ratios $A_{1} / A_{0}$ and $A_{2} / A_{0}$ diverge for large attraction $(\beta V \ll 0)$.

For large repulsive lateral interactions $(\beta V \gg 0)$ and coverage $\theta<1 / 2$, one obtain the following limits $A_{1} / A_{0} \rightarrow-1$ and $A_{2} / A_{0} \rightarrow 1$ (this is because $\langle\bullet \bullet\rangle \rightarrow 0$ ). Then, the sticking coefficient is given by Eq. (47).

For $\theta>1 / 2, A_{1} / A_{0} \rightarrow 0$ and the expression for the normalized sticking coefficient is given by

$$
\frac{S(\theta, T)}{S(0, T)}=\frac{(1-\theta)(2 \theta-1)^{2}}{\left[2 \theta-1+e^{\beta U} \sqrt{\theta(1-\theta)}\right]\left[2 \theta-1+e^{-\beta U} \sqrt{\theta(1-\theta)}\right]} .
$$

The TPD spectra for the TDA scheme are similar to the Ising kinetics. This behavior is valid provided that $U<V$. However, for $U \gg V$, the TPD spectra for mobile adsorbate present a different behavior with respect to the corresponding to immobile adsorbate. In fact, in Eq. (49) the main contribution to the transition probabilities is due to $U$. Particularly, for repulsive lateral interaction the immobile TPD curves have only two peaks instead of three, as is observed in Fig. 5.

\section{Standard Glauber dynamics (hard dynamics)}

This dynamic scheme is one of the most well known and widely used; there are several cases that can be cited, for instance, very recently it has been implemented to analyze the distribution of gold and palladium atoms on $\mathrm{Au} / \mathrm{Pd}(111)$ alloys [63]. The approach to equilibrium is ensured by a single-spin-flip (nonconservative) dynamics which satisfies detailed balance, such as the Metropolis or Glauber algorithms $[19,20,53]$. Any such algorithm applied to adsorptiondesorption processes is defined by the following transition probabilities: 

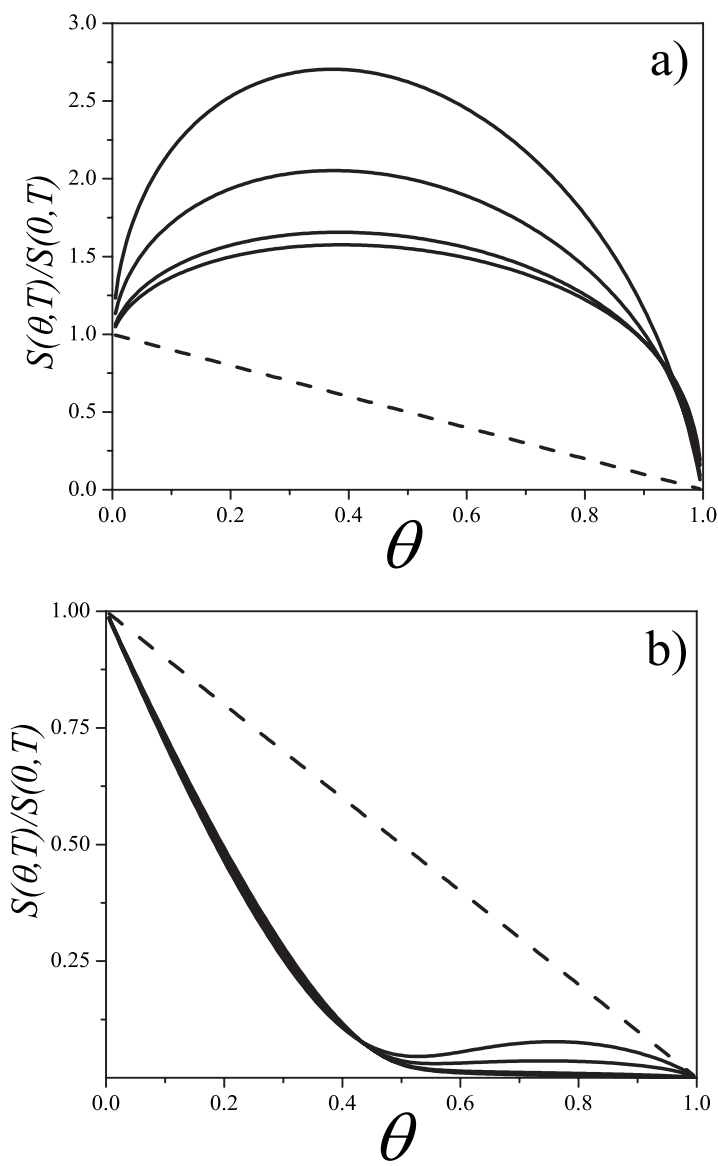

FIG. 4. Normalized sticking coefficient for TDA with different values of parameter $U$. (a) Attractive $(\beta V=-4)$ and (b) repulsive $(\beta V=4)$ lateral interactions. In both figures, $\beta U=0,2,4,10$ from top to bottom at $\theta=0.6$. The sticking coefficient for $V=0$ (dash) is also included.

$$
W_{a d s}^{i}=\left(\frac{1}{1+e^{\beta\left(E_{S}+i V\right)}}\right)
$$

and

$$
W_{d e s}^{i}=\left(\frac{1}{1+e^{-\beta\left(E_{\left.S^{+i V}\right)}\right.}}\right) .
$$

The corresponding adsorption and desorption coefficients are given by

$$
A_{i}=\left(\frac{1}{1+e^{\beta\left(E_{S}+i V\right)}}\right)-\sum_{j=0}^{i} j A_{j-1}
$$

and

$$
D_{i}=\left(\frac{1}{1+e^{-\beta\left(E_{S}+i V\right)}}\right)-\sum_{j=0}^{i} j D_{j-1},
$$

respectively.

The adsorption isotherms, the sticking coefficients and TPD spectra calculated with the standard Glauber dynamics present the same behavior that the corresponding to the Ising

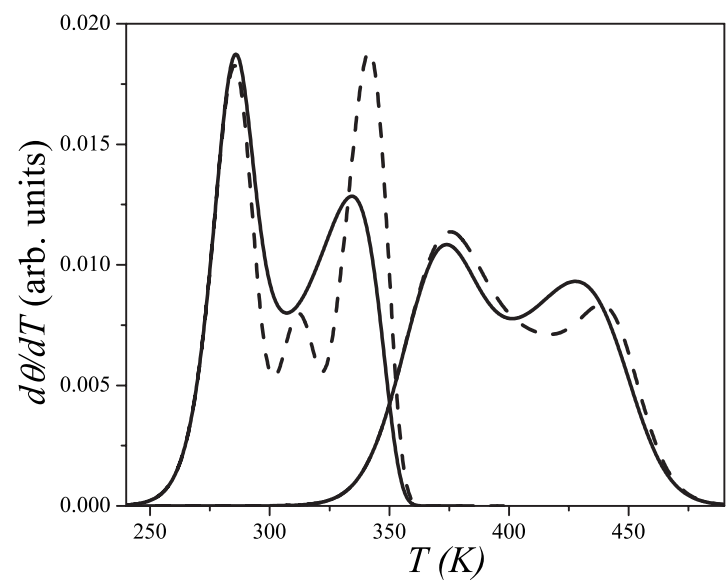

FIG. 5. Mobile (line) and immobile (dash) TPD spectra using TDA for repulsive lateral interaction, $V=2 \mathrm{kcal} / \mathrm{mol}$ and two different values of parameter $U: U<2 \mathrm{kcal} / \mathrm{mol}$ (left) and $U$ $=20 \mathrm{kcal} / \mathrm{mol}$ (right).

kinetics with $\gamma=-1$. In particular, the ratio between the adsorption coefficients $A_{1} / A_{0}$ and $A_{2} / A_{0}$ in both schemes are the same.

\section{Interaction kinetics (soft dynamics)}

This scheme was introduced by Payne and Kreuzer $[17,18,21,24]$. It is based on the following relation between the adsorption and desorption coefficients:

$$
\frac{A_{i}}{A_{0}}=\gamma \frac{D_{i}}{D_{0}},
$$

where $\gamma$ is a proportionality coefficient and $A_{0}=1$. The PDB imposes that

$$
\begin{gathered}
D_{1}=D_{0}\left(\frac{e^{\beta V}-1}{1-\gamma e^{\beta V}}\right), \\
D_{2}=D_{0}\left(\frac{e^{2 \beta V}-1}{1-\gamma e^{2 \beta V}}\right)-2 D_{1}
\end{gathered}
$$

and

$$
D_{0}=e^{\beta E_{S}} .
$$

These relations are equivalent to the following transition probabilities:

$$
W_{a d s}^{i}=\gamma \frac{(1-\gamma)}{\left(e^{-\beta i V}-\gamma\right)}
$$

and

$$
W_{d e s}^{i}=e^{\beta E_{S}} \frac{(1-\gamma)}{\left(e^{-\beta i V}-\gamma\right)} .
$$

According to the discussion given by Manzi et al. [30] it is easy to see that Eqs. (19)-(24) impose new restrictions on the $A_{i}$ and $D_{i}$ coefficients. Considering these additional restrictions certain values of $\gamma$ are not allowed. These forbidden values depend on the lateral interaction $V$ and they are 


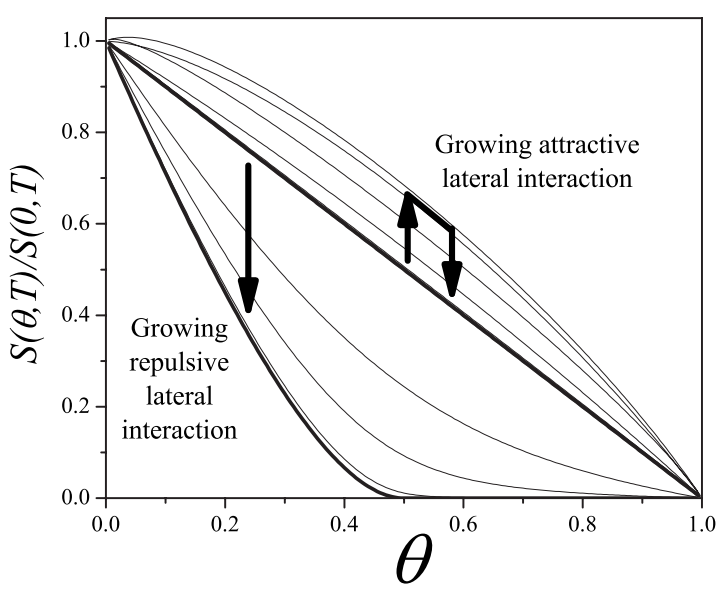

FIG. 6. Normalized sticking coefficients for interaction kinetics with $\gamma=-1$ and different values of the lateral interaction.

shown in a phase diagram [Fig. 1(a)] in Ref. [30].

The adsorption isotherms calculated for those values of $\gamma$ and $V$ belonging to region I are well-behaved. For those values of $\gamma$ out of this region, the adsorption isotherms have kinks at nonzero temperatures. Since the adsorption isotherms are equilibrium properties, this would indicate that this dynamics, as well as Ising kinetics, do not satisfy the principle of detailed balance. However, as it is pointed out in Ref. [30], the PDB is not enough to guarantee the correct behavior of the equilibrium and nonequilibrium quantities, and additional constrains are necessary. In particular for those values of $\gamma$ belonging to the forbidden region, the relations given in Eqs. (19)-(24) are not fulfilled and consequently the behavior of the observables will be anomalous.

In Fig. 6, the normalized sticking coefficient is shown as a function of the surface coverage with $\gamma=-1$. As it is observed, for repulsive lateral interactions, $V>0$, the curves are below $(1-\theta)$. This behavior is well discussed in the literature $[3,14,18,17,21,24]$. Particularly, Kreuzer in Ref. [18] analyzed, in the framework of the interaction kinetics, the coverage and temperature dependence of the sticking coefficient in the presence of intrinsic and extrinsic precursor states with different lateral interactions.

Note that when $\beta V \gg 0$, the ratios $A_{1} / A_{0} \rightarrow-1$ and $A_{2} / A_{0} \rightarrow 1$, independently of $\gamma$. Therefore, for $\theta<1 / 2$, the sticking coefficient is given by Eq. (47), while for $\theta>1 / 2$, $S(\theta, T) / S(0, T) \rightarrow 0$. However, the main feature appears for attractive lateral interactions $(V<0)$. In this case, the sticking coefficient increases reaching a limit value, and then diminishes until reaching the limit $(1-\theta)$. Up to now, this result has not been discussed in the literature. To explain this behavior, note that when $\beta V \ll 0,\langle\bullet \bullet\rangle \rightarrow \theta$ [see Eq. (27) or Eq. (28)], $A_{1} / A_{0} \rightarrow-\gamma$ and $A_{2} / A_{0} \rightarrow \gamma$. Consequently, the last two terms in rhs of Eq. (34) vanish and the normalized sticking coefficient goes to $(1-\theta)$, which coincides with the one corresponding to $V=0$.

The TPD spectra are analyzed in what follows. For attractive lateral interactions the behavior is similar to the Ising kinetics. However, for repulsive lateral interactions, the behavior of the immobile and mobile TPD curves are certainly anomalous for finite values of $\gamma$ (see Fig. 7). The tempera-

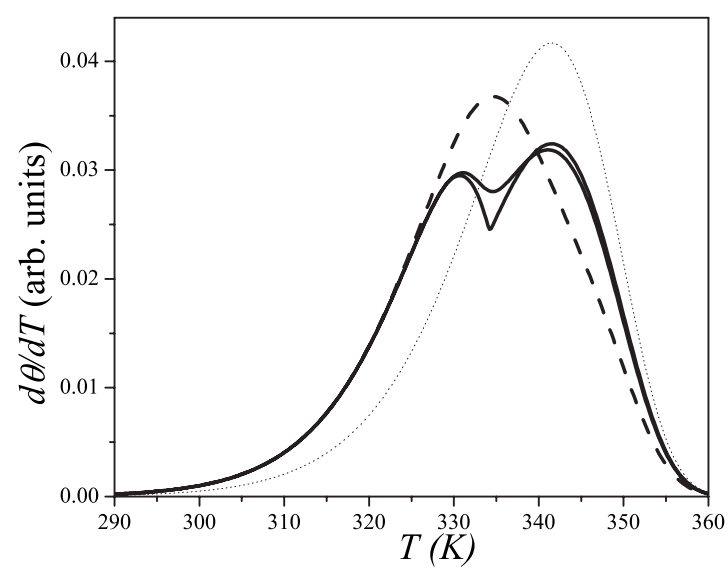

FIG. 7. Thermal desorption spectra using interaction kinetics with $\gamma=-1$ for repulsive lateral interaction. $V=0$ (dot), immobile (dash) TPD spectra $V=3 \mathrm{kcal} / \mathrm{mol}$ and mobile (solid) TPD spectra $V=3,10 \mathrm{kcal} / \mathrm{mol}$.

ture separation of the two peaks, corresponding to the mobile TPD, does not depend on the lateral interaction. On the other hand the three peaks in the immobile TPD can be obtained only in the limit $\gamma \rightarrow 0$.

\section{E. Transition state theory (soft dynamics)}

The transition state theory (TST) provides a way to obtain the constant rates of the involved processes through the knowledge of the appropriate kinetic equation $[14,30,64-69]$. The expressions for the transition probabilities in the framework of the TST are [30] the following:

$$
W_{a d s}^{i}=e^{-\beta\left(E_{S} / 2+\epsilon_{i}^{*}\right)}
$$

and

$$
W_{d e s}^{i}=e^{\beta\left(E_{S} / 2-\left(\epsilon_{i}^{*}-\epsilon_{i}\right)\right)} .
$$

Then, the corresponding adsorption and desorption coefficients are given by

$$
\begin{gathered}
A_{0}=e^{-\beta\left(E_{S} / 2+\epsilon_{0}^{*}\right)}, \\
A_{1}=e^{-\beta E_{S} / 2}\left(e^{-\beta \epsilon_{1}^{*}}-e^{-\beta \epsilon_{0}^{*}}\right), \\
A_{2}=e^{-\beta E_{S} / 2}\left(e^{-\beta \epsilon_{2}^{*}}-2 e^{-\beta \epsilon_{1}^{*}}+e^{-\beta \epsilon_{0}^{*}}\right), \\
D_{0}=e^{\beta\left[E_{S^{\prime}} 2-\left(\epsilon_{0}^{*}-\epsilon_{0}\right)\right]}, \\
D_{1}=e^{\beta E_{S} / 2}\left(e^{-\beta\left(\epsilon_{1}^{*}-\epsilon_{1}\right)}-e^{-\beta\left(\epsilon_{0}^{*}-\epsilon_{0}\right)}\right)
\end{gathered}
$$

and

$$
D_{2}=e^{\beta E_{S} / 2}\left(e^{-\beta\left(\epsilon_{2}^{*}-\epsilon_{2}\right)}-2 e^{-\beta\left(\epsilon_{1}^{*}-\epsilon_{1}\right)}+e^{-\beta\left(\epsilon_{0}^{*}-\epsilon_{0}\right)}\right),
$$

where $\epsilon_{i}$ and $\epsilon_{i}^{*}$ are the lateral interactions (with $i$ neighbors) in the initial (ground) and activated (transition) states. These interactions can be calculated using density functional theory (DFT) [70-78]. 


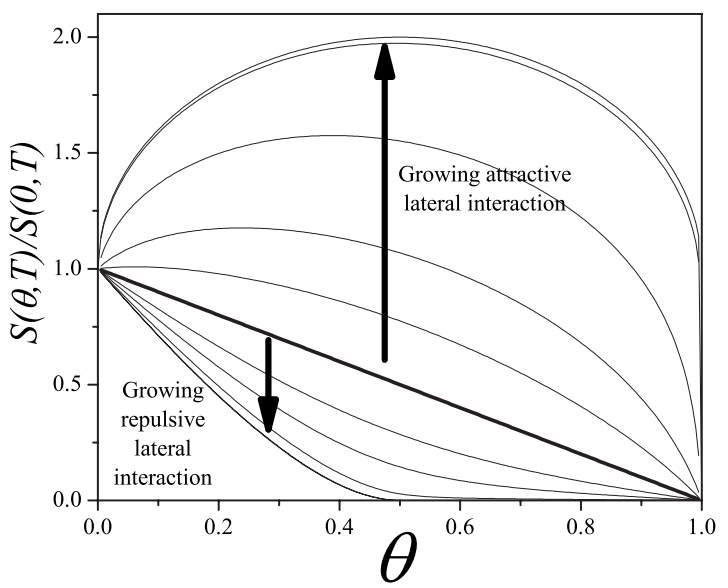

FIG. 8. Normalized sticking coefficients for TST with BrönstedPolanyi relations for different values of the lateral interaction.

The PDB imposes that

$$
\epsilon_{i}=i V
$$

In many cases, TST can be combined with the BrönstedPolanyi-type relations between the lateral interactions in the ground and activated states [79], for instance:

$$
\epsilon_{i}^{*}=\frac{i V}{2} .
$$

Then, one can obtain the following expressions for the $A$ and $D$ coefficients:

$$
A_{i}=\exp \left[-\beta E_{s} / 2\right](\exp [-\beta V / 2]-1)^{i}
$$

and

$$
D_{i}=\exp \left[\beta E_{s} / 2\right](\exp [\beta V / 2]-1)^{i},
$$

with $i=0,1,2$ for the one-dimensional case.

A detailed analysis of the sticking coefficient for the TST is given in Ref. [30]. In Fig. 8, the sticking coefficient for the TST is shown (combined with the Brönsted-Polanyi-type relations). As it is observed, for large repulsive interactions, the sticking coefficient behaves as in the interaction kinetics. However, for attractive lateral interactions, the sticking coefficient increases monotonically for large lateral interactions. This is because the ratios $A_{1} / A_{0} \rightarrow \infty$ and $A_{2} / A_{0} \rightarrow \infty$ when $\beta V \ll 0$.

Due to the fact that TST does not impose any restriction on the value and sign of the lateral interaction in both, the ground and activated states, several scenarios can be obtained for the desorption processes. It is well known that the immobile TPD for high initial coverages present three peaks $[80,81]$. However, the presence of three peaks in the TPD curves for immobile particles is not caused only by repulsive lateral interactions. In fact, a TPD curve with three peaks can be obtained with $\epsilon_{i}^{*}<0$ and $\epsilon_{i}<0$, while the sticking coefficient for these interactions is higher than the Langmuir's sticking coefficient. On the other hand, a TPD spectra with only one peak for repulsive lateral interactions, with a peak temperature higher than for the noninteracting case can be obtained [30].

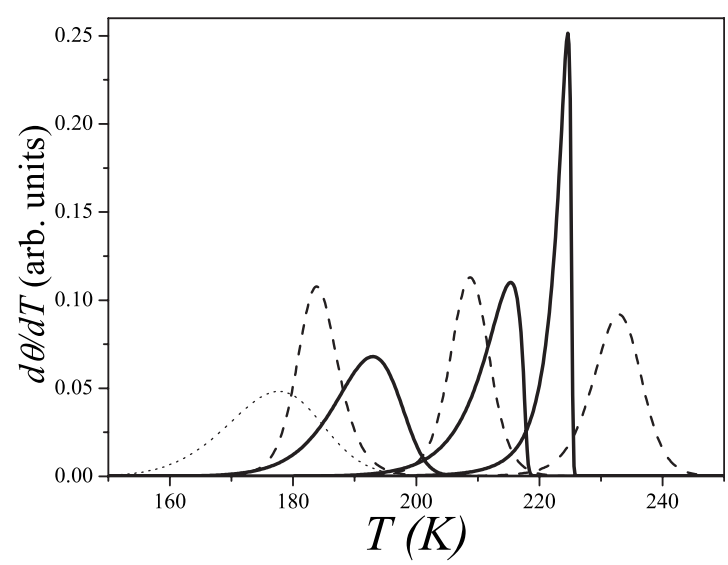

FIG. 9. Thermal desorption spectra using TST for attractive lateral interaction $V=2 \mathrm{kcal} / \mathrm{mol}$ and different values of the lateral interaction in activated state. The case $V=0$ (dot) is also included. Immobile (dash) TPD spectra: left to right $\epsilon_{1}^{*}=V, V / 2,0$; and mobile (solid) TPD spectra: left to right $\epsilon_{1}^{*}=V, V / 2,0$. Here $\epsilon_{2}^{*}=2 \epsilon_{1}^{*}$ is considered

Another particularity of the TST spectra is that both the mobile and immobile TPD curves shift to lower temperatures as soon as $\epsilon^{*} \rightarrow \epsilon$ for a fixed value of attractive lateral interaction, $V<0$. This effect can be explained by considering the desorption probability, where the activated state lateral interaction shields the effect of the lateral interaction, $V$. On the other hand, the peak of the TPD curve, corresponding to the immobile adsorbate, moves from higher to lower temperatures with respect to the mobile adsorbate, as it is observed in Fig. 9.

\section{F. Soft Glauber dynamics (soft dynamics)}

In the soft Glauber dynamics the adsorption and desorption probabilities are defined as

$$
W_{a d s}^{i}=\left(\frac{e^{-\beta E_{S}}}{1+e^{-\beta E_{S}}}\right)\left(\frac{e^{-\beta i V}}{1+e^{-\beta i V}}\right)
$$

and

$$
W_{d e s}^{i}=\left(\frac{e^{\beta E_{S}}}{1+e^{\beta E_{S}}}\right)\left(\frac{e^{\beta i V}}{1+e^{\beta i V}}\right),
$$

respectively. The corresponding adsorption coefficients are the following:

$$
\begin{gathered}
A_{0}=\left(\frac{1}{1+e^{\beta E_{S}}}\right), \\
A_{1}=\frac{-e^{\beta V}}{\left(1+e^{\beta E_{S}}\right)\left(1+e^{\beta V}\right)},
\end{gathered}
$$

and

$$
A_{2}=\frac{2 e^{\beta V}-e^{\beta 2 V}+e^{\beta 3 V}}{\left(1+e^{\beta E_{S}}\right)\left(1+e^{\beta 2 V}\right)\left(1+e^{\beta V}\right)} .
$$

Similarly for the desorption coefficients, 


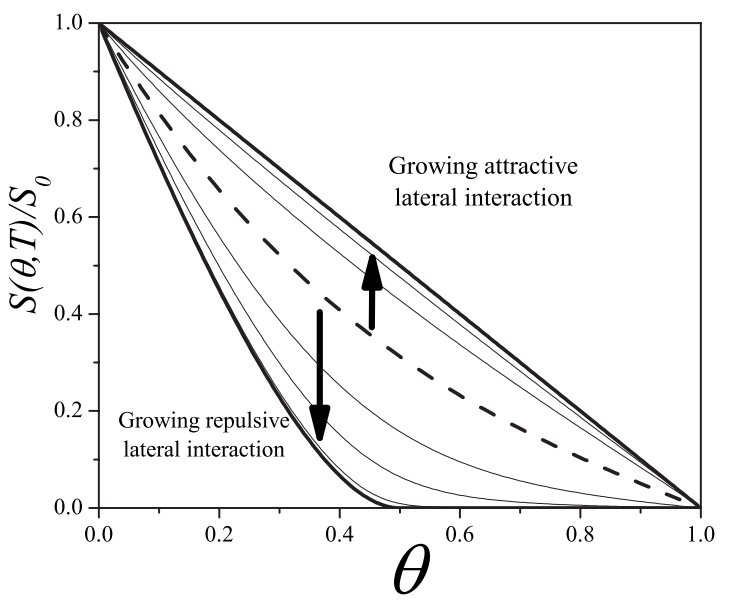

FIG. 10. Normalized sticking coefficients for soft Glauber dynamics for different values of the lateral interaction.

$$
\begin{gathered}
D_{0}=\left(\frac{1}{1+e^{-\beta E_{S}}}\right), \\
D_{1}=\frac{-e^{-\beta V}}{\left(1+e^{-\beta E_{S}}\right)\left(1+e^{-\beta V}\right)},
\end{gathered}
$$

and

$$
D_{2}=\frac{2 e^{-\beta V}-e^{-\beta 2 V}+e^{-\beta 3 V}}{\left(1+e^{-\beta E_{S}}\right)\left(1+e^{-\beta 2 V}\right)\left(1+e^{-\beta V}\right)} .
$$

The normalized sticking coefficient for the soft Glauber dynamics is shown in Fig. 10. For attractive lateral interactions, the sticking coefficient presents an upper limit given by $S(\theta, T) / S(0, T)=(1-\theta)$. This is because, $A_{1} / A_{0} \rightarrow 0$ and $A_{2} / A_{0} \rightarrow 0$ when $\beta V \ll 0$.

For large repulsion $(\beta V \gg 0)$ and $\theta<1 / 2, A_{1} / A_{0} \rightarrow-1$ and $A_{2} / A_{0} \rightarrow 1$, then the normalized sticking coefficient is given by Eq. (47), while for $\theta>1 / 2$, . Note that, for $V=0$, the ratios are $A_{1} / A_{0}=-1 / 2$ and $A_{2} / A_{0}=1 / 2$, consequently the corresponding sticking curve is given by

$$
\frac{S(\theta, T)}{S(0, T)}=1-2 \theta+\frac{3}{2} \theta^{2}-\frac{1}{2} \theta^{3} .
$$

The TPD spectra for attractive lateral interactions do not present any particularity. However, they do for null and repulsive lateral interactions. These spectra are shown in Fig. 11. As it is observed for $V>0$, the mobile and immobile TPD curves present almost the same behavior even for large repulsion. On the other hand, for null interaction, the mobile and immobile TPD curves do not coincide.

\section{G. Inverse relation (soft dynamics)}

Other example of symmetric relation between the adsorption and desorption coefficients is introduced by considering the following relations:

$$
A_{i}=e^{-\beta\left(E_{S} / 2+i V\right)}
$$

and

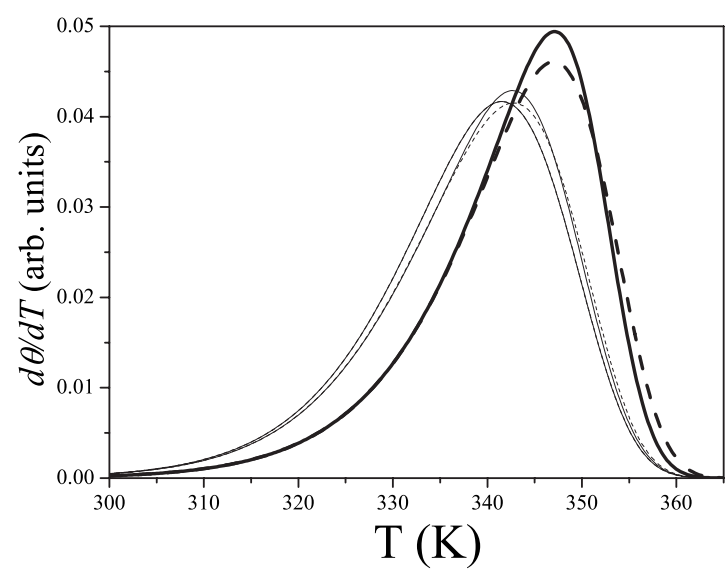

FIG. 11. Immobile (dash) and mobile (solid) thermal desorption spectra using soft Glauber dynamics for repulsive and noninteracting lateral interaction. Left to right $V(\mathrm{kcal} / \mathrm{mol})=10,1,0$.

$$
D_{i}=e^{\beta\left(E_{S} / 2+i V\right)},
$$

for $i=0,1,2$. This is equivalent to consider the following transition probabilities:

$$
W_{a d s}^{i}=e^{-\beta E_{S} / 2}\left(1+e^{-\beta V}\right)^{i}
$$

and

$$
W_{d e s}^{i}=e^{\beta E_{S} / 2}\left(1+e^{\beta V}\right)^{i} .
$$

The normalized sticking coefficients versus coverage are shown in Fig. 12. As it is observed, the normalized sticking coefficient tends to the limit curve $(1-\theta)$, for large repulsion. In fact, when $\beta V \gg 0$, the ratios $A_{1} / A_{0} \rightarrow 0$ and $A_{2} / A_{0} \rightarrow 0$. Note that, for $V=0$ the ratios are $A_{1} / A_{0}=1$ and $A_{2} / A_{0}=1$. Therefore, the corresponding sticking curve is given by

$$
\frac{S(\theta, T)}{S(0, T)}=1+\theta-\theta^{2}-\theta^{3} .
$$

On the other hand, for attractive lateral interactions $(V<0)$ the sticking coefficient presents the usual behavior, growing

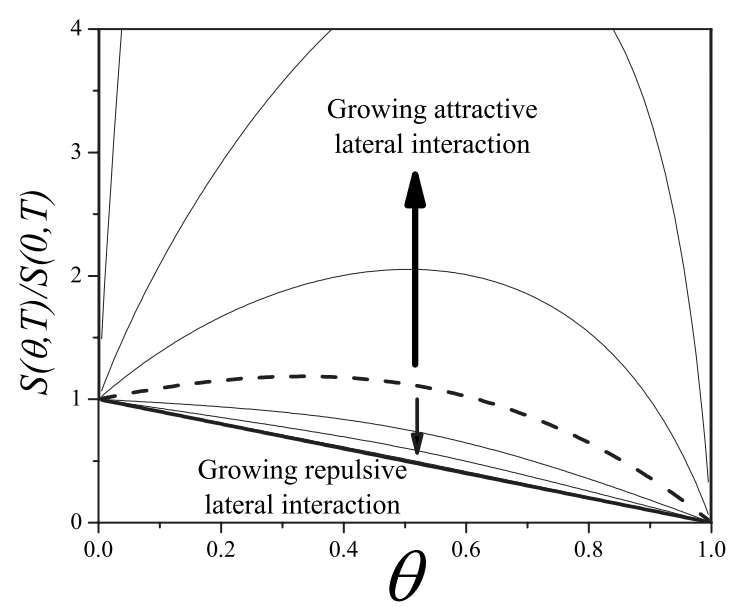

FIG. 12. Normalized sticking coefficient for Inverse relation where different values of lateral interaction are considered. The sticking coefficient for $V=0$ (dash) is also included. 


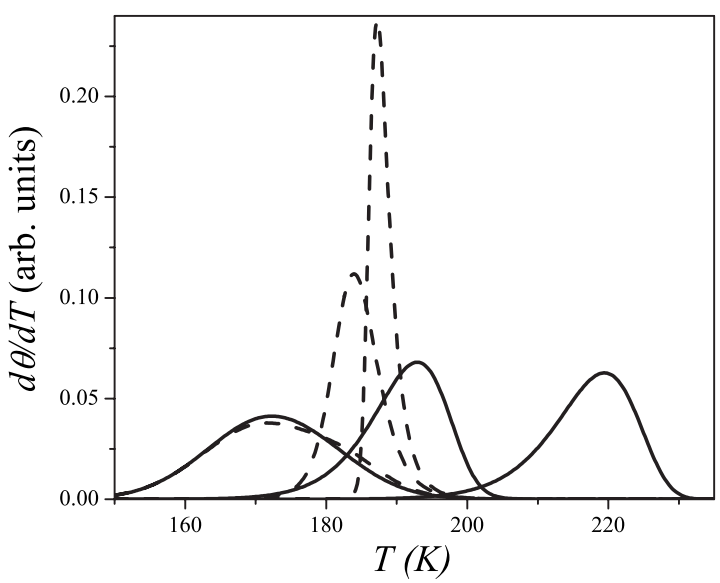

FIG. 13. Thermal desorption spectra using inverse relation for different values of the attractive and null lateral interaction. Immobile (dash) TPD spectra: left to right $V(\mathrm{kcal} / \mathrm{mol})=0,-2,-6$; and mobile (solid) TPD spectra: left to right $V(\mathrm{kcal} / \mathrm{mol})=0,-2,-6$.

with increasing interaction. For large attraction, $\beta V \ll 0$, both $A_{1} / A_{0}$ and $A_{2} / A_{0}$ diverge. Then, the sticking coefficient increases monotonically in the range $0<\theta<1$.

In this case, the behavior of the TPD spectra for repulsive lateral interaction is similar to the Ising kinetics. However, for attractive lateral interactions, the behavior of the mobile and immobile TPD spectra is different from the cases analyzed above. In fact, the shifting of the peaks as a function of lateral interactions is smaller for the immobile TPD spectra, compared to the mobile case. Moreover, for null interaction, the mobile TPD spectra do not coincide with the immobile (see Fig. 13).

\section{H. One-step dynamics (soft dynamics)}

In the one-step dynamics (OSD) $[48,49]$ the expressions for the transition probabilities of the adsorption and desorption processes are given by

$$
W_{a d s}^{i}=e^{-\beta U} e^{-\beta\left(E_{S}+i V\right) / 2}
$$

and

$$
W_{d e s}^{i}=e^{-\beta U} e^{\beta\left(E_{S^{+}}+i\right) / 2},
$$

respectively. The parameter $U$ has the same meaning that in TDA. After some algebra, the following expressions for the adsorption and desorption coefficients can be obtained:

$$
\begin{gathered}
A_{0}=e^{-\beta U} \exp \left[-\beta E_{s} / 2\right], \\
A_{1}=e^{-\beta U} \exp \left[-\beta E_{s} / 2\right](\exp [-\beta V / 2]-1), \\
A_{2}=e^{-\beta U} \exp \left[-\beta E_{s} / 2\right](\exp [-\beta V / 2]-1)^{2}, \\
D_{0}=e^{-\beta U} \exp \left[\beta E_{s} / 2\right], \\
D_{1}=e^{-\beta U} \exp \left[\beta E_{s} / 2\right](\exp [\beta V / 2]-1),
\end{gathered}
$$

and
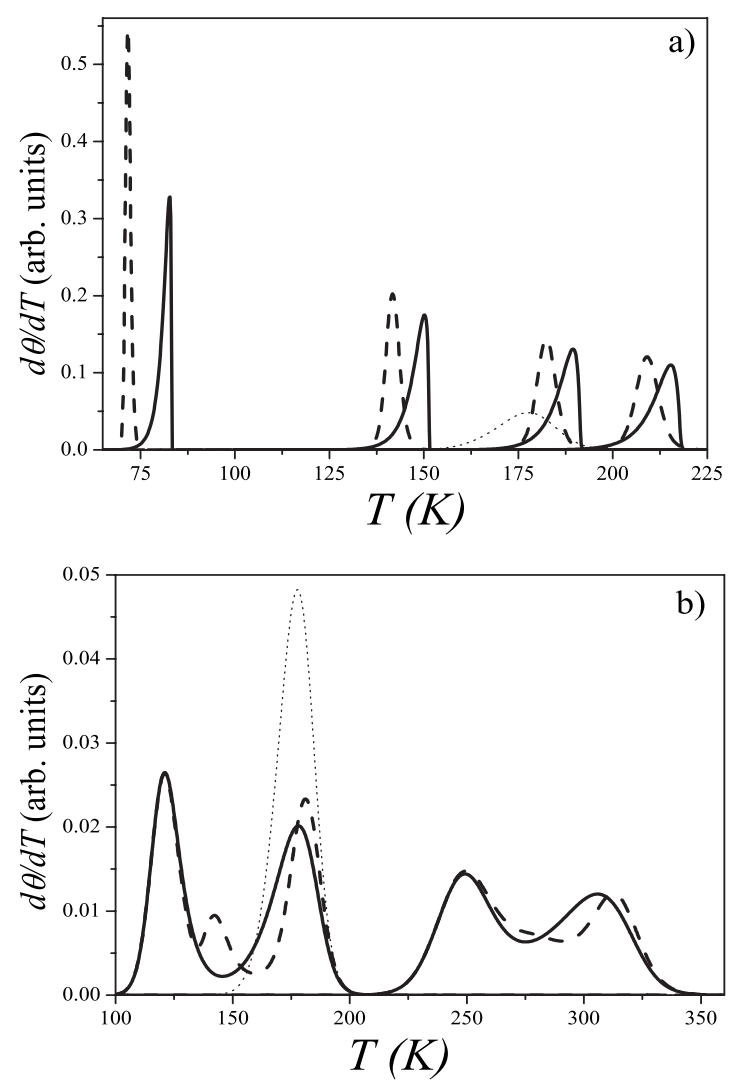

FIG. 14. Thermal desorption spectra using OSD for different values of the parameter $U$. (a) attractive $V=-2 \mathrm{kcal} / \mathrm{mol}$ lateral interaction, immobile (dash) and mobile (solid) TPD spectra: left to right $U(\mathrm{kcal} / \mathrm{mol})=10,5,2,0$; (b) repulsive $V=2 \mathrm{kcal} / \mathrm{mol}$ lateral interaction, immobile (dash) and mobile (solid) TPD spectra: $U(\mathrm{kcal} / \mathrm{mol})=0$ (left), 10 (right). In both figures $V=0$ (dot) is also included.

$$
D_{2}=e^{-\beta U} \exp \left[\beta E_{s} / 2\right](\exp [\beta V / 2]-1)^{2} .
$$

The OSD can be reduced to the Brönsted-Polanyi-type TST dynamics setting $U=0$.

The sticking coefficient for the OSD behaves similar to the one corresponding to the TST. It is easy to verify that the sticking coefficient does not depend on $U$.

One of the main features observed in the OSD is the influence of the transition state energy $U$ on the TPD spectra. In fact, for attractive lateral interactions, the TPD curves move to lower temperatures with respect to the noninteracting desorption curve for increasing values of $U$ (see, Fig. 14). On the contrary, for repulsive lateral interaction, the TPD curves shift to higher temperatures than the ones corresponding to the noninteracting curve. Moreover, in the last case, the middle peak of the TPD curve disappears for increasing values of $U$.

\section{SUMMARY AND CONCLUSION}

The aim of this work is the study of the influence of the dynamic scheme in the adsorption-desorption kinetics. The analytical treatment has been done in a one-dimensional system, mainly due to two reasons. The first one is the avail- 
ability of the exact solutions for the coverage and two-site correlation function (which is not possible in higher dimensions). The second one, is the presence of phase transitions in two and higher dimensions. This could mask the possible anomalous behaviors in the observables, which is precisely the objective of the present investigation.

A complete kinetic description should contemplate certain coherence in the behavior of the observables.

Since adsorption isotherms are equilibrium properties, all dynamic schemes (hard and soft) give the same results. However, for interaction kinetics and Ising kinetics, restrictions on the lateral interaction and the parameter $\gamma$ have been found. Out of the range of validity, the adsorption isotherms have shown kinks at nonzero temperatures for both dynamic schemes. Certainly, this behavior has no physical meaning in one-dimensional systems with short-range interactions.

The normalized sticking coefficient is calculated for all dynamic schemes. The limits for large interactions (attractive and repulsive), as well as for $V=0$, are exactly obtained in several cases.

The hard dynamic schemes, Glauber, Ising, and TDA (provided that $V$ and $U$ are of the same magnitude) give a well-behaved normalized sticking coefficient. In other word, it increases with attractive lateral interactions and drops to zero at monolayer coverage, while for repulsive lateral interactions it decreases below the line $1-\theta$, which corresponds to null interaction. The only difference between Ising and TDA occurs for repulsive lateral interactions and $\theta>1 / 2$, where for Ising kinetics the sticking coefficient is not null, while for TDA it can be null for higher values of $U$.

In the soft dynamic schemes, with the exception of the TST and OSD, the normalized sticking coefficient is not well behaved. In fact, for the interaction kinetics the sticking coefficient increases reaching a limiting value and then diminishes until reaching the limit $(1-\theta)$. On the other hand, for the inverse relation and soft Glauber dynamics the normalized sticking coefficient for null interaction, $V=0$, does not correspond to $1-\theta$. In fact, a large repulsion gives this limit in the interaction kinetics scheme, while a large attraction is necessary to reach this limit in the soft Glauber dynamics scheme.

In order to compare both desorption regimens, the immobile TPD spectra are exactly calculated using a $(3,2)$ cluster approximation, while the mobile TPD spectra are obtained by using the phenomenological equation [Eq. (39)] which is valid for very fast diffusion.

The hard dynamic schemes give a well-behaved desorption spectra, namely, starting from high enough initial coverage and repulsive lateral interactions the TPD curves for immobile adsorbate must present the characteristic three peaks, while the mobile TPD must present two peaks. On the other hand, for attractive lateral interactions the TPD spectra present only one peak that shifts to higher temperatures with increasing interactions.

In the case of Ising kinetics, the only restriction is imposed on the free parameter. The standard Glauber dynamic is a particular case of the Ising kinetics. On the other hand, in the TDA the energy barrier between the final and initial states cannot take an arbitrary value.

On the contrary, the soft dynamic schemes present several problems. The exceptions are the TST and the OSD. However, in the first one, the lateral interaction of the activated states must be carefully chosen, according to the lateral interaction in the ground states. For the OSD, the restriction is similar to the TDA.

For example, for the hard dynamic schemes with attractive lateral interaction the peak of the mobile TPD occurs at lower temperatures than for the immobile TPD, while for the soft dynamic schemes, this behavior is not observed in all the cases. In fact, this effect is the opposite for the OSD, while for the TST it depends on the relation between the values of $\epsilon_{i}^{*}$ and $\epsilon_{i}$.

Then, it can be concluded that the principle of detailed balance is a necessary but not sufficient condition to guarantee the consistency in the kinetics. As it is demonstrated in this work, the hard dynamic schemes and the transition state theory show a good consistency between the three observables, while most of the soft dynamic schemes present several problems, some of which can be considered as anomalous behaviors.

Finally, this paper can be used as a guide to define a realistic kinetic Monte Carlo algorithm, where the large kinetic and structural effects can arise from seemingly minor modifications of the transition rates.

\section{ACKNOWLEDGMENTS}

We specially thank to Dr. A. J. Boscoboinik for reading the manuscript. This work was partially supported by the CONICET (Argentina).
[1] Frontier in Surface and Interface Science, edited by C. B. Duke and E. W. Plummer (North-Holland, Amsterdam, 2002).

[2] Handbook of Heterogeneous Catalysis, edited by G. Ertl, H. Knozinger, and J. Weitkamp (Wiley, New York, 1997).

[3] A. Cassuto and D. A. King, Surf. Sci. 102, 388 (1981).

[4] A. Córdoba and M. C. Lemos, J. Chem. Phys. 99, 4821 (1993).

[5] J. W. Evans, Rev. Mod. Phys. 65, 1281 (1993) and references therein.
[6] D. Menzel, in Interactions on Metal Surfaces, edited by R. Gomer (Springer-Verlag, Berlín, 1975), p. 102.

[7] Y. K. Tovbin, in Equilibria and Dynamics of Gas Adsorption on Heterogeneous Solid Surfaces, Studies in surface science and catalysis, Vol. 104, edited by W. Rudzinski, A. Steele, and G. Zgrablich (Elsevier, New York, 1997) and references therein.

[8] V. P. Zhdanov and B. Kasemo, Chem. Phys. 177, 519 (1993) and references therein. 
[9] H. J. Kreuzer and Z. W. Gortel, Physisorption Kinetics (Springer-Verlag, Berlin, 1986).

[10] H. J. Kreuzer and S. H. Payne, Surf. Sci. 198, 235 (1988).

[11] H. J. Kreuzer and S. H. Payne, Surf. Sci. 200, L433 (1988).

[12] S. H. Payne and H. J. Kreuzer, Surf. Sci. 205, 153 (1988).

[13] C. Uebing and V. P. Zhdanov, J. Chem. Phys. 109, 3197 (1998).

[14] V. P. Zhdanov, Elementary Physicochemical Processes on Solid Surfaces (Plenum, New York, 1991).

[15] M. Silverberg and A. Ben-Shaul, Surf. Sci. 214, 17 (1989).

[16] S. J. Lombardo and A. T. Bell, Surf. Sci. Rep. 13, 3 (1991).

[17] H. J. Kreuzer and S. H. Payne, in Equilibria and Dynamics of Gas Adsorption on Heterogeneous Solid Surfaces, Studies in Surfaces Science and Catalysis, Vol. 104, edited by W. Rudzinski, W. A. Steele, G. Zgrablich (Elsevier, New York, 1997). p. 153, and references therein.

[18] H. J. Kreuzer, J. Chem. Phys. 104, 9593 (1996).

[19] R. J. Glauber, J. Math. Phys. 4, 294 (1963).

[20] K. Kawasaki, in Phase Transitions and Critical Phenomena, edited by C. Domb and M. S. Green (Academic Press, New York, 1972), Vol. 2, p. 443.

[21] H. J. Kreuzer and J. Zhang, Appl. Phys. A: Mater. Sci. Process. 51, 183 (1990).

[22] S. H. Payne, H. A. McKay, H. J. Kreuzer, M. Gierer, H. Bludau, H. Over, and G. Ertl, Phys. Rev. B 54, 5073 (1996).

[23] S. H. Payne, G. Ledue, J. C. Michael, and H. J. Kreuzer, Surf. Sci. 512, 151 (2002).

[24] S. H. Payne, H. J. Kreuzer, M. Kinne, R. Denecke, and H.-P. Steinruck, Surf. Sci. 513, 174 (2002).

[25] G. Costanza, S. Manzi, and V. D. Pereyra, Surf. Sci. 524, 89 (2003).

[26] O. Furlong, S. Manzi, G. Costanza, and V. D. Pereyra, Physica A 339, 267 (2004).

[27] G. Costanza, S. Manzi, and V. D. Pereyra, Surf. Sci. 600, 3484 (2006).

[28] S. H. Payne and H. J. Kreuzer, Phys. Rev. B 75, 115403 (2007).

[29] A. Wierzbicki and H. J. Kreuzer, Surf. Sci. 257, 417 (1991).

[30] S. J. Manzi, R. E. Belardinelli, G. Costanza, and V. D. Pereyra, Phys. Rev. E 79, 021103 (2009).

[31] O. Shochet, K. Kassner, E. Ben-Jacob, S. B. Lipson, and H. Müller-Krümbhaar, Physica A 181, 136 (1992) and references therein.

[32] D. P. Landau and K. Binder, Monte Carlo Simulations in Statistical Physics (Cambridge University Press, Cambridge, 2000).

[33] M. Siegert and M. Plischke, Phys. Rev. E 50, 917 (1994).

[34] Y. Shim and D. P. Landau, Phys. Rev. E 64, 036110 (2001).

[35] P. A. Rikvold and M. Kolesik, J. Stat. Phys. 100, 377 (2000).

[36] P. A. Rikvold and M. Kolesik, J. Phys. A 35, L117 (2002).

[37] P. A. Rikvold and M. Kolesik, Phys. Rev. E 66, 066116 (2002).

[38] P. A. Rikvold and M. Kolesik, Phys. Rev. E 67, 066113 (2003).

[39] G. M. Buendía, P. A. Rikvold, and M. Kolesik, Phys. Rev. B 73, 045437 (2006); J. Mol. Struct.; THEOCHEM 769, 207 (2006).

[40] J. Marro and R. Dickman, Nonequilibrium Phase Transitions in Lattice Model (Cambridge University Press, Cambridge, 1999), Chap. 7.
[41] S. Frank, D. E. Roberts, and P. A. Rikvold, J. Chem. Phys. 122, 064705 (2005).

[42] K. Saito, S. Takesue, and S. Miyashita, Phys. Rev. E 61, 2397 (2000).

[43] K. Park and M. A. Novotny, Comput. Phys. Commun. 147, 737 (2002).

[44] G. M. Buendía, P. A. Rikvold, M. Kolesik, K. Park, and M. A. Novotny, Phys. Rev. B 76, 045422 (2007).

[45] S. Frank and P. A. Rikvold, Surf. Sci. 600, 2470 (2006).

[46] G. M. Buendía, P. A. Rikvold, K. Park, and M. A. Novotny, J. Chem. Phys. 121, 4193 (2004).

[47] K. Park, P. A. Rikvold, G. M. Buendía, and M. A. Novotny, Phys. Rev. Lett. 92, 015701 (2004).

[48] K. A. Fichthorn and W. H. Weinberg, J. Chem. Phys. 95, 1090 (1991).

[49] H. C. Kang and W. H. Weinberg, J. Chem. Phys. 90, 2824 (1989).

[50] T. Ala-Nissila and S. C. Ying, Prog. Surf. Sci. 39, 227 (1992).

[51] T. Ala-Nissila, R. Ferrando, and S. C. Ying, Adv. Phys. 51, 949 (2002).

[52] T. Ala-Nissila, J. Kjoll, and S. C. Ying, Phys. Rev. B 46, 846 (1992).

[53] K. Kawasaki, Phys. Rev. 145, 224 (1966), ; 148, 375 (1966); 150, 285 (1966).

[54] H. J. Kreuzer and S. H. Payne, Computational Methods in Colloid and Interface Science (Dekker, New York, 1999).

[55] S. H. Payne, A. Wierzbicki, and H. J. Kreuzer, Surf. Sci. 291, 242 (1993).

[56] D. ben-Avraham and J. Köhler, Phys. Rev. A 45, 8358 (1992).

[57] J. Evans, D. K. Hoffman, and H. Pak, Surf. Sci. 192, 475 (1987).

[58] S. H. Payne and H. J. Kreuzer, Surf. Sci. 222, 404 (1989).

[59] F. Haake, M. Leeuweinstein, and M. Wilkens, Z. Phys. B 54, 333 (1984); 55, 211 (1984).

[60] J. H. Luscombe, Phys. Rev. B 29, 5128 (1984).

[61] E. Oguz, O. T. Valls, G. F. Mazenko, and J. H. Luscombe, Surf. Sci. 118, 578 (1981).

[62] D. J. W. Geldart, H. J. Kreuzer, and F. S. Rys, Surf. Sci. 176, 284 (1986).

[63] J. A. Boscoboinik, C. Plaisance, M. Neurock, and W. T. Tysoe, Phys. Rev. B 77, 045422 (2008).

[64] V. P. Zhdanov, Surf. Sci. 111, 63 (1981); 111, L662 (1981); 123, 106 (1982); 133, 469 (1983); 157, L384 (1985); 165, L31 (1986); 171, L461 (1986); 209, 523 (1989).

[65] V. P. Zhdanov, Surf. Sci. Rep. 12185 (1991) and reference therein.

[66] V. D. Pereyra and G. Zgrablich, Langmuir 6, 118 (1990).

[67] V. D. Pereyra, G. Zgrablich, and V. P. Zhdanov, Langmuir 6, 691 (1990).

[68] B. Li, C.-S. Zhang, V. P. Zhdanov, and P. R. Norton, Surf. Sci. 322, 373 (1995).

[69] V. P. Zhdanov and B. Kasemo, Surf. Sci. 412, 527 (1998).

[70] P. Piercy, K. De'Bell, and H. Pfnür, Phys. Rev. B 45, 1869 (1992).

[71] C. Schwennicke and H. Pfnür, Phys. Rev. B 56, 10558 (1997).

[72] V. P. Zhdanov and T. Matsushima, Phys. Rev. Lett. 98, 036101 (2007).

[73] R. Kose, W. A. Brown, and D. A. King, J. Phys. Chem. 103, 8722 (1999).

[74] C. Stampfl, H. J. Kreuzer, S. H. Payne, H. Pfnür, and M. 
Scheffler, Phys. Rev. Lett. 83, 2993 (1999).

[75] E. Hansen and M. Neurock, Surf. Sci. 441, 410 (1999).

[76] J.-S. McEwen and A. Eichler, J. Chem. Phys. 126, 094701 (2007).

[77] A. P. J. Jansen and W. K. Offermans, J. Comput. Methods Sci. Eng. 2, 351 (2002)

[78] C. G. M. Hermse, F. Frechard, A. P. van Bavel, J. J. Lukkien, J. W. Niemantsverdriet, R. A. van Santen, and A. P. J. Jansen,
J. Chem. Phys. 118, 7081 (2003).

[79] R. A. van Santen and J. W. Niemantsverdriet, Chemical Kinetics and Catalysis (Plenum Press, New York, 1995).

[80] J. L. Sales and G. Zgrablich, Phys. Rev. B 35, 9520 (1987); Surf. Sci. 187, 1 (1987).

[81] J. L. Sales, R. O. Uñac, M. V. Gargiulo, V. Bustos, and G. Zgrablich, Langmuir 12, 95 (1996). 